\title{
Permanent Magnets Agingn in Variable Flux Permanent Magnet Synchronous Machines
}

\author{
Daniel Fernández ${ }^{1}$, Maria Martinez ${ }^{1}$, David Reigosa ${ }^{1}$ Juan Guerrero $^{1}$, Carlos Suarez $^{2}$, Fernando Briz ${ }^{1}$ \\ ${ }^{1}$ Dept. of Elect., Computer \& System Engineering \\ ${ }^{2}$ Construction and Fabrication Engineering Department \\ University of Oviedo, Gijón, Spain \\ fernandezalodaniel@uniovi.es, martinezgmaria@uniovi.es, diazdavid@uniovi.es, guerrero@uniovi.es, csuarez@uniovi.es, \\ fernando@isa.uniovi.es
}

\begin{abstract}
Permanent magnet synchronous machines (PMSMs) operation above based speed is typically achieved by injecting negative $d$-axis current to produce flux weakening. However, this increases permanent magnet (PM) losses, penalizing the efficiency and increasing the risk of demagnetization. Variable Flux PMSMs (VF-PMSM) in which PMs are magnetized/demagnetized during normal operation of the drive have been proposed to avoid the use of flux weakening current. However, aging effects due to PM magnetization/demagnetization cycles have not thoroughly studied yet. This paper analyzes the variation of $\mathrm{NdFeB}$ PMs properties (i.e. magnetic, electric and thermal properties) resulting from magnetization/demagnetization cycles. ${ }^{1}$
\end{abstract}

Keywords-Permanent magnet aging, re-magnetization, variable flux permanent magnet synchronous machine.

\section{INTRODUCTION}

Efficient and safe operation of electric drives can be especially challenging for applications in which the machine must operate at high speed. High speed operation of conventional PMSMs, i.e. interior PMSMs (IPMSMs) and surface PMSMs (SPMSMs), require the injection of negative current in the $d$-axis, also known as field weakening current [1]. Flux weakening current reduces the stator flux linkage matching the back-EMF with the available voltage from the inverter. However, flux weakening current also increases the airgap flux harmonic content [2], [3], and consequently eddy-currents in PMs and stator/rotor cores, reducing the efficiency and increasing temperature and consequently, the risk of PM demagnetization [3].

To avoid the injection of flux weakening current and the subsequent adverse effects, variable flux PMSMs (VFPMSM, also called memory motors) have been developed. In this type of machines, the PMs magnetization state (MS) is changed during normal operation [4], [5].

VF-PMSMs can be equipped with different magnet types, including low coercivity magnets (low-Hc) like

1 This work was supported in part by the Research, Technological Development and Innovation Programs of the Spanish Ministry Economy and Competitiveness, under grant MINECO-17-ENE2016-80047-R.
AlNiCo [6],[7] or SmCo [8], or a combination of low- and high-Hc magnets [3],[8]-[10]. VF-PMSMs equipping AlNiCo magnets require relatively small magnetic fields to change the MS [4], [6], [7]; this limits machine torque capability as the magnetic field produced by the $q$-axis current could demagnetize the PMs [8], [10]. VF-PMSMs combining low- and high-Hc magnets reduce the risk of undesired demagnetization due to $q$-axis current. In this configuration high-Hc magnets provide the base rotor magnetic field, which is unaffected by current, while $d$-axis current pulses are used to changes the MS of low-Hc magnets. In all cases, VF-PMSMs low-Hc magnets must be magnetized/demagnetized as required, depending on the working condition of the machine.

In-situ magnetization of PMs has been widely investigated [6]-[12]. In-situ magnetizers, typically capacitor-discharge impulse based, are devices independent of the machine used in industry mass production [12]: once the rotor lamination is assembled, demagnetized PMs are inserted in their corresponding slots; an in-situ magnetizer is then used to magnetize the PMs for the final machine assembling. Remagnetization of PMSMs after partial demagnetization resulting from a fault condition (e.g. over temperature or short-circuit current) can be accomplished if an in-situ magnetizer is used [12], but the machine needs to be disassembled. In VF-PMSM, in-situ magnetization is achieved by injecting a short current pulse from the stator terminals, i.e. de machine does not have to be disassembled [10]-[11]. VF-PMSMs MS can be manipulated thousands of times all along its life [6]; aging effects on PMs due to magnetization/ demagnetization cycling cycles has not been performed yet.

This paper analyzes aging effects on PM magnetic, electric and thermal properties, due to magnetization /demagnetization cycles. Long-term behavior (i.e. degradation) of magnetic properties of PMs used in VFPMSMs has been studied using magnetized samples under different working conditions [13]-[15].

The paper is organized as follows: characterization of PMs is discussed in section II, the test setup designed to cycle and characterize PMs is analyzed in Section III, experimental results using magnet samples are provided in 
TABLE I. MAGNETIC, ELECTRIC AND THERMAL PROPERTIES OF TYPICAL MAGNETS

\begin{tabular}{|c|c|c|c|c|c|c|c|c|}
\hline Magnet Type & $\mathrm{Br}(\mathrm{T})$ & $\alpha_{\mathrm{B}}(\% / \mathrm{K})$ & $\mathrm{H}_{\mathrm{c}}(\mathrm{kA} / \mathrm{m})$ & $\mathrm{H}_{\text {cJ }}(\mathrm{kA} / \mathrm{m})$ & $\alpha_{\mathrm{H}}(\% / \mathrm{K})$ & $\mathrm{BH}_{\max } \mathrm{kJ} / \mathrm{m}^{3}$ & $\rho 10^{-6}(\Omega \mathrm{m})$ & $\mathrm{T}_{\max }\left({ }^{\circ} \mathrm{C}\right)$ \\
\hline Ferrite 27/25SH & 0.400 & -0.2 & 278 & 302 & +0.3 & 30 & 104 & 250 \\
\hline AlNiCo Cast 80/12 & 1.11 & $-0.01 \sim-0.035$ & 130 & 126 & $-0.03 \sim 0.03$ & 80 & $0.45-0.55$ & 500 \\
\hline SmCo YXS24 & 1.02 & -0.035 & 764 & 2000 & -0.25 & 191 & $0.75-0.85$ & 250 \\
\hline NdFeB N42SH & 1.33 & $-0.11 \sim-0.12$ & 1018 & 1595 & $-0.56 \sim-0.70$ & 334 & $1.4-1.6$ & 150 \\
\hline
\end{tabular}

*Source: [15]

Section IV, and conclusions are finally provided in Section VI.

\section{CHARACTERIZATION OF PERMANENT MAGNETS}

This section describes the main magnetic, electric and thermal properties that characterize PM materials and that will be considered therefore for the study of aging.

- PM magnetic properties can be obtained from the hysteresis loop, i.e. a plot of magnetic induction, B, and magnetic polarization, $\mathrm{J}$, as a function of the magnetic field strength, $\mathrm{H}$ [16].

- PM thermal properties can be estimated from the analysis of the hysteresis loop at different temperatures [16].

- $\quad$ PM electrical properties can be estimated applying a time varying field to the PM [18].

Table I shows the main properties of four different PM types used in PMSMs: AlNiCo, ferrite, SmCo and NdFeB; where $\mathrm{Br}$ accounts the flux density of the magnet in a closed magnetic circuit without externally applied field, $H_{C J}$ is the intrinsic coercivity that measures the magnet's resistance to demagnetization, $B H_{\max }$ is the maximum energy product, i.e. an metric of the energy available for interaction within a magnetic circuit, $\rho$ is the electrical resistivity, $T_{\max }$ is the maximum working temperature, $\alpha_{B}$ is the reversible thermal coefficient of $B r$ and $\alpha_{H}$ is the reversible thermal coefficient of $H_{c J}$.

a)

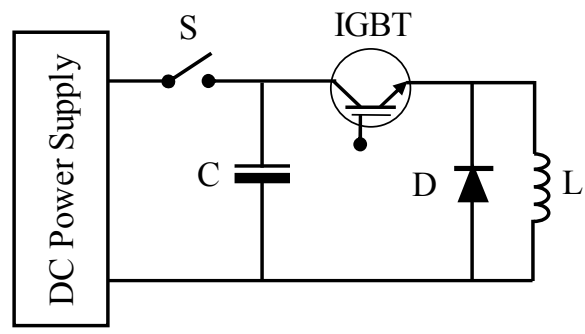

b)

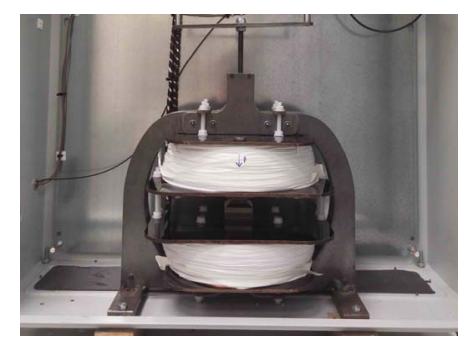

Fig. 1. Schematic representation of the impulse magnetizer electric circuit, a), and picture of the magnetizer, $b$ ).

The magnetic measurements shown in this paper follow the methods described in IEC60404-05:2015 standard and refer to the measurements in a closed magnetic circuit.

\section{TEST SETUP}

This section describes the setup used to analyze PMs aging effects. A capacitor-discharge impulse magnetizer is used to magnetize magnet samples according to IEC604045. The PM material is saturated by the field induced by the discharging current of the capacitors. Hysteresis loop of hard magnetic material must be obtained from previously magnetized samples. Fig. 1 shows a schematic representation and a picture of the peak magnetizer, its main characteristics are given in Table II. The hysteresis loop is measured using a hysteresis graph as described in the standard, from which all magnetic properties listed in section II can be obtained; PM magnet resistance is estimated from its induced eddy currents. Fig. 2 shows the schematic of the setup used for BH characterization of PMs according to IEC 60404-5 standard. The iron core is made of laminated soft magnetic material; its central column being movable to match PM size. The test samples have a cylindrical shape (see Fig. 4) and regular end faces as recommended by the standard. Once the samples are prepared, the magnetizer is controlled to magnetize/demagnetize the magnet sample repeatedly, accelerating the aging process.

TABLE II: PEAK MAGNETIZER CHARACTERISTICS

\begin{tabular}{|c|c|}
\hline PARAMETER & \\
\hline Maximum voltage & $730 \mathrm{~V}$ \\
\hline Maximum energy & $3500 \mathrm{~J}$ \\
\hline Maximum airgap (With poles) & $60 \mathrm{~mm}$ \\
\hline Maximum airgap (without poles) & $20 \mathrm{~mm}$ \\
\hline Maximum field & $2200 \mathrm{kA} / \mathrm{m}$ \\
\hline
\end{tabular}

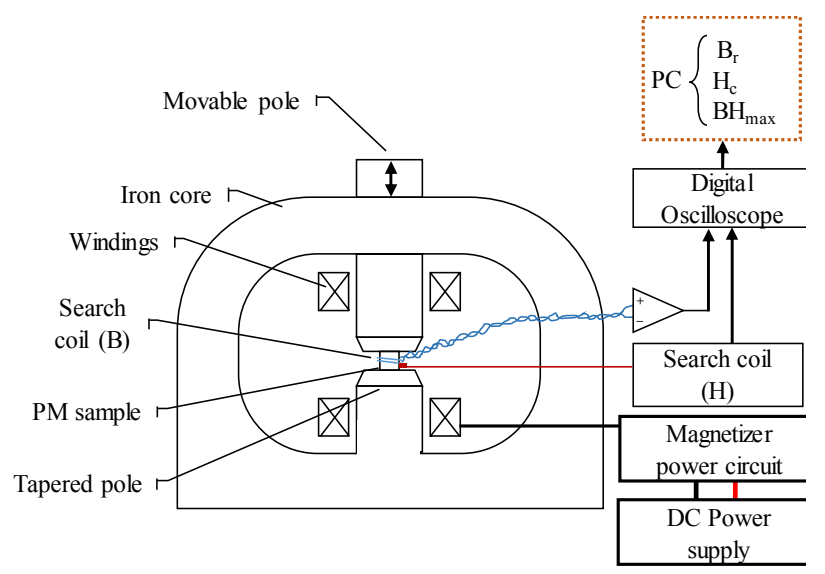

Fig. 2. Setup for BH curve measurement according to IEC 60404-5 st andard. 


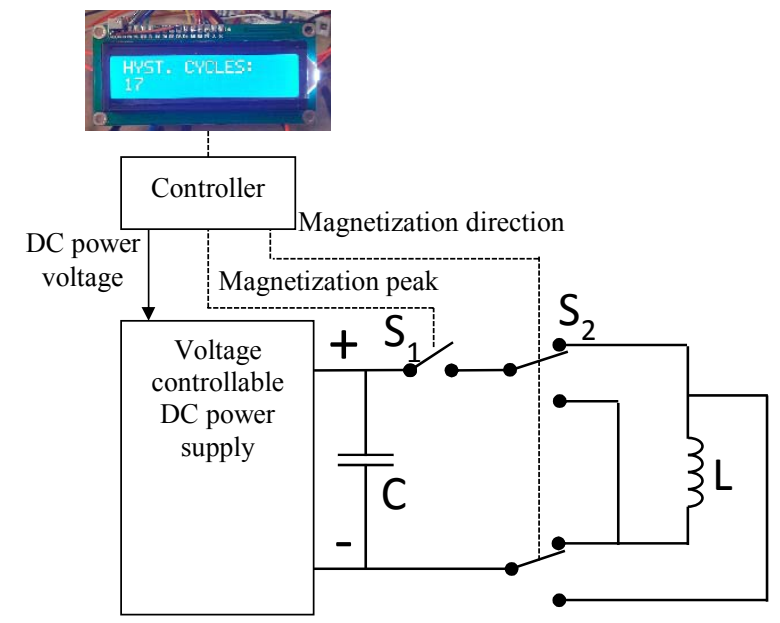

Fig. 3. Peak magnetizer electric circuit, a), and yoke, b).

Two search coils, see Figs. 2 and 6, are used to measure the magnetic flux density (B), and the magnetic field strength $(\mathrm{H})$; Fig. 5a and $5 \mathrm{~b}$ shows the induced voltages in the two search coils during a remagnetization process; Fig. 5c and 4d showing the resulting magnetic flux density and magnetic field strength. Changes in the PM magnetic flux density, $\Delta B$ (1), are obtained by integrating the voltage induced in " $B$ " coil, $U$ (see Fig. 5a and $4 \mathrm{c}$ ), where $B_{2}$ and $B_{1}$ are the magnetic flux densities in the instant $t_{2}$ and $t_{1}, A$ is the cross-section area of the PM sample and $N$ is the number of turns of the search coil. The magnetic field strength, $H(2)$, is obtained from the voltage induced in " $H$ " coil, see Figs. $4 \mathrm{~b}$ and $4 \mathrm{~d}$, which must be placed within a homogeneous field in the airgap close to the PM sample, according to IEC60404-05. Sample and coil axes must be aligned. The demagnetization curve of the PM is usually represented as $\mathrm{B}-\mathrm{H}$ or J-H, $J$ being the polarization, obtained from (3).

$$
\begin{gathered}
\Delta B=B_{2}-B_{1}=\frac{1}{A N} \int_{t_{1}}^{t_{2}} U d t \\
\Delta H=H_{2}-H_{1}=\frac{1}{\mu_{0} A N} \int_{t_{1}}^{t_{2}} U d t \\
J=B-\mu_{0} H
\end{gathered}
$$

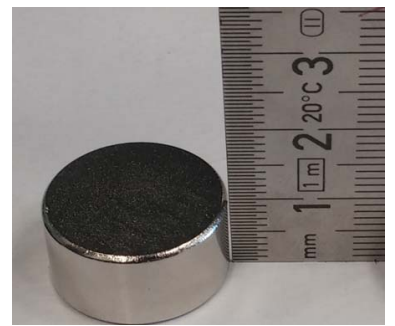

Fig. 4. NdFeB test sample, $20 \mathrm{~mm}$ diameter and $10 \mathrm{~mm}$ height.
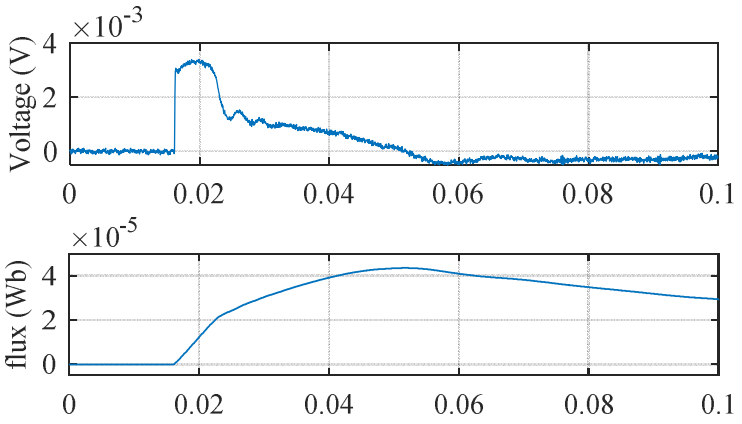

b)

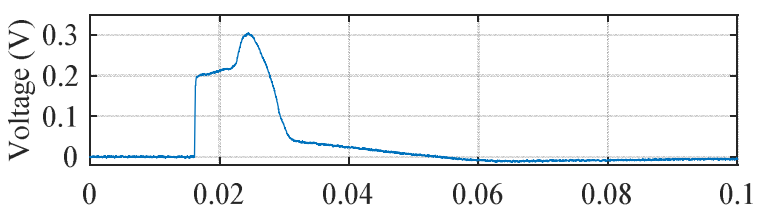

c)

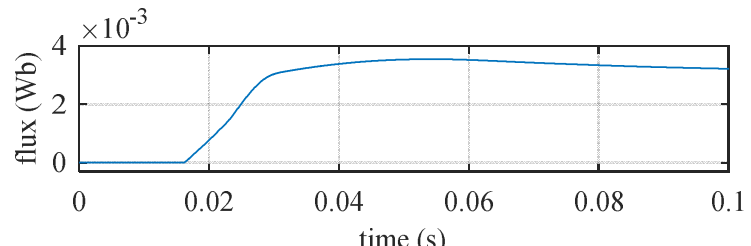

d)

Fig. 5. Induced voltage in B coil, a), and in $\mathrm{H}$ coil, b) and resulting flux in $\mathrm{B}$ coil, c), and $\mathrm{H}$ coil, $\mathrm{d}$ ), during a remagnetization process.

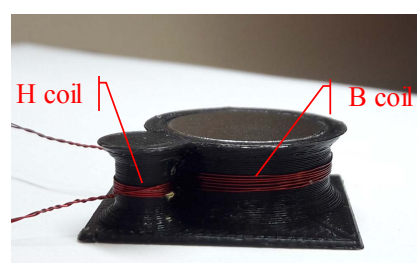

Fig. 6. Search coils for $\mathrm{H}$ and $\mathrm{B}$ measurements.

PM resistivity could be also a metric of the PM aging. PM resistance can be estimated by applying a time varying field to the PM [18]-[19], the frequency must be chosen to avoid sking effect and full penetration of the high frequency current in the PM material; the system shown in Fig. 7 has been used for this purpose [18]-[19]. It consists of a core made of iron powder blocks, to minimize Eddy current losses, a coil and an adjustable airgap to fit the PM size. The coil is feed from an H-bridge converter able to inject a high frequency signal of different frequencies and amplitudes into the coil, which will be used to induce a high frequency flux through the PM. The resulting high frequency Eddy currents in the PM will depend on its resistivity. The PM resistivity will be estimated from the coil voltages and currents [18]-[19].
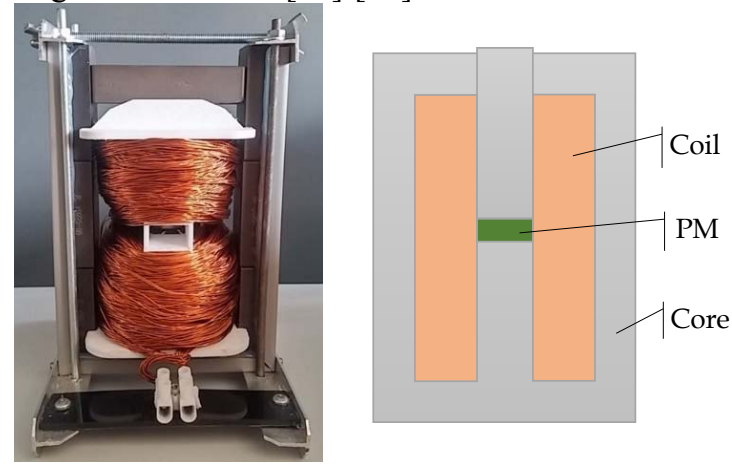

Fig. 7. Experimental setup used for PM resistance evaluation 


\section{EXPERIMENTAL RESULTS}

Experimental results using $\mathrm{NdFeB}$ PMs samples (se Fig. 4) have been conducted. Fig. 8 shows the PM flux measured in an open magnetic circuit for a constant PM temperature vs. number of hysteresis cycles. It can be observed from Fig. 8 that PM surface flux density initially decreases as the number of cycles increases, remaining constant when the of cycles is $>2000$. Fig. 9 shows the demagnetization curve of the PM at the beginning and end of the aging process shown in Fig. 8. It can be observed that both $\mathrm{B}-\mathrm{H}$ and $\mathrm{J}-\mathrm{H}$ curves have been negatively affected as the curves have shrunk. Table IV shows the PM magnetic properties before and after the aging process; It can be observed that all magnetic properties $\left(\mathrm{Br}, \mathrm{H}_{\mathrm{cJ}}, \mathrm{H}_{\mathrm{c}}\right.$ and $\left.\mathrm{BH}_{\max }\right)$ are worsen due to magnet cycling: $\mathrm{Br}, \mathrm{HcJ}, \mathrm{Hc}$ and $\mathrm{BH}_{\max }$ have decreased by $9,8 \%, 3,4 \%, 10 \%$ and $20 \%$ respectively.

Consequences of this degradation in the machine performance include a reduction of torque capability due to $\mathrm{Br}$ and $\mathrm{BH}_{\max }$ decrease, increased risk of demagnetization as a reduction of $\mathrm{Hc}$ and $\mathrm{HcJ}$ may lead to irreversible demagnetization for $q$-axis currents, and an efficiency decrease as larger currents levels will be needed to produce a given torque.

PM high frequency resistance has also been evaluated using the system shown in Fig. 7, the results are shown in Table IV. The increase of the resistance $\approx 6.5 \%$ in Table IV is consistent with the results shown in Fig. 10, as PM high frequency resistance is a reliable indicator of the PM MS [19], as PM Eddy current losses are proportional to the PM high frequency resistance [20].

Consequences of HF resistivity increase of a PM in the machine performance include a PM Eddy current losses increase and therefore, PM temperature increase [18]; resulting in a torque, power density and efficiency reduction, and in an increased risk of demagnetization.

Table IV: PM Properties before and after remagnetization

\begin{tabular}{|l|l|l|l|}
\hline & 0 cycles & 5250 cycles & Variation \\
\hline $\mathrm{Br}(\mathrm{T})$ & 1.344 & 1.223 & $-9.8 \%$ \\
\hline $\mathrm{BH}_{\max }\left(\mathrm{kJ} / \mathrm{m}^{3}\right)$ & 320 & 265 & $-20 \%$ \\
\hline $\mathrm{H}_{\mathrm{c}}(\mathrm{kA} / \mathrm{m})$ & -961 & -868 & $-10 \%$ \\
\hline $\mathrm{H}_{\mathrm{cJ}}(\mathrm{kA} / \mathrm{m})$ & -1885 & -1823 & $-3.4 \%$ \\
\hline Estimated HF resistance $(\Omega)$ & 0.854 & 0.909 & $+6.5 \%$ \\
\hline
\end{tabular}

Temperature properties of cycled PMs have also been evaluated. Fig. 10a shows the PM flux measured in an open magnetic circuit for different PM temperatures. PM flux is seen to decrease with the number of cycles, which is consistent with the results shown in Fig. 10a. It is also observed from Fig. 10a that the knee point, which corresponds to the maximum working temperature, does not change with cycling. Fig. 10b shows the flux density difference between a non-cycled and a cycled PM vs. magnet temperature; it is observed that the slightly increases with temperature, i.e. PM flux temperature dependence increases due to repeated cycling. This represents a change in reversible thermal coefficient of remanence, $\alpha_{\mathrm{Br}}$, that represents the change of PM remanence with temperature.

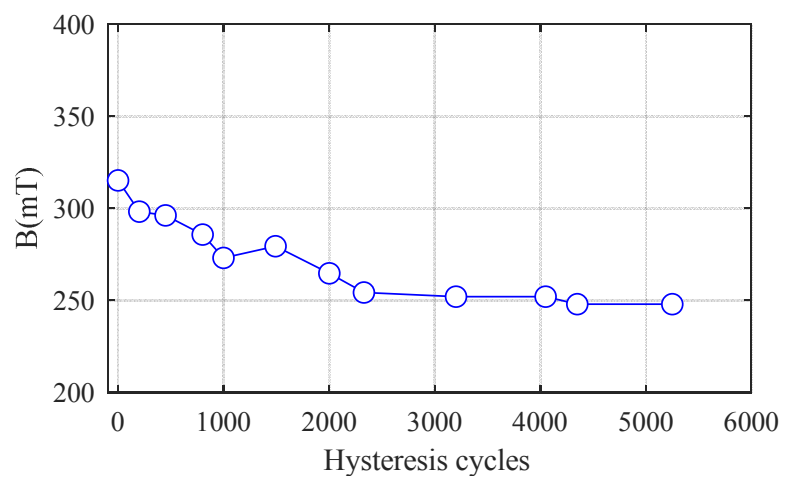

Fig. 8. PM flux density measured at the center of the PM's surface under open magnetic circuit conditions. Constant PM temperature, $20^{\circ} \mathrm{C}$.

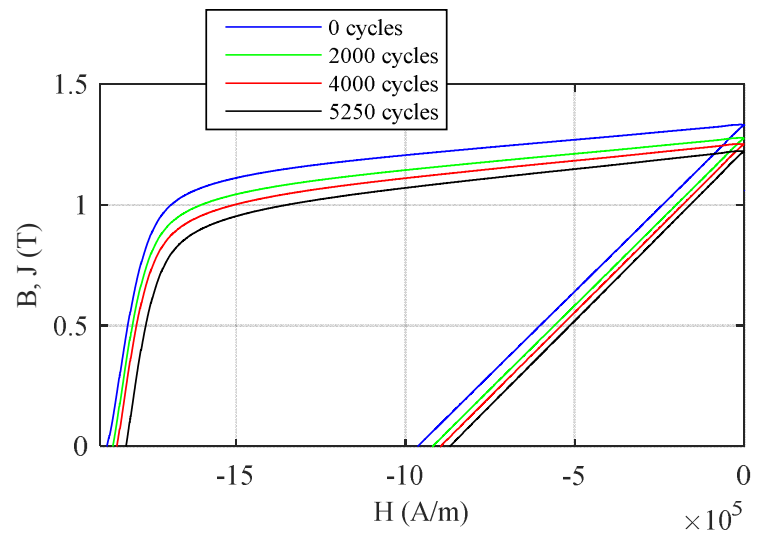

Fig. 9. BH curve before and after 5250 hysteresis cycles. Constant PM t emperature, $20^{\circ} \mathrm{C}$.
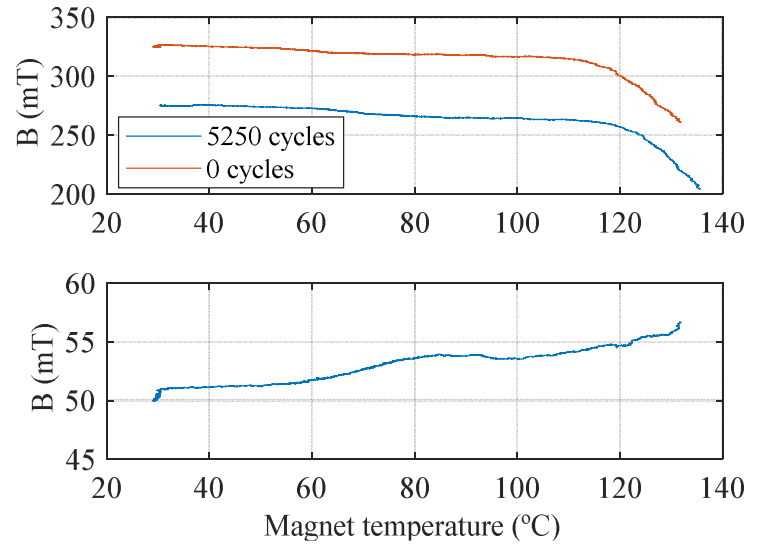

Fig. 10. PM flux variation with temperature (open circuit) and b) PM flux d ifference between non-cycled and cycled PM (curves in Fig. 10a).

Finally, PM surface flux density distribution of a cycled PMs was evaluated in an open magnetic circuit using a Gauss meter. Fig 11 shows the magnetic flux density measured all along the diameter $(D)$ of the PM, see Fig. 12, before and after cycling it. It is observed that the minimum magnetic flux density for both, cycled and non-cycled PM, occurs at the PM center; the variation of the magnetic flux density being higher for the cycled than for the non-cycled PM.

The consequences of a non-uniform magnetic flux density distribution in a VF-PMSM are additional harmonic 
components in the MMF waveform. This will lead to higher core losses and lower machine performance.

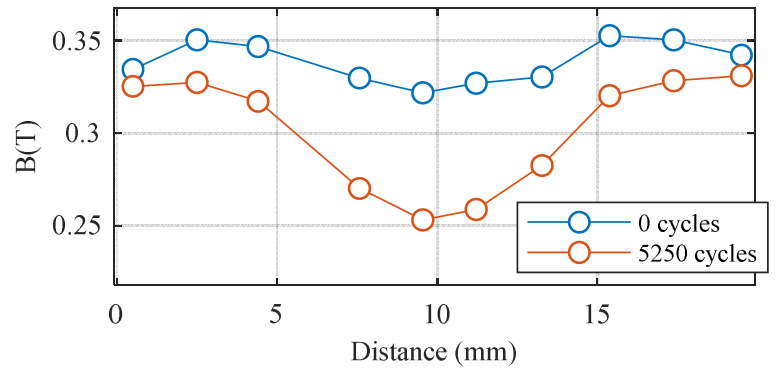

Fig. 11. PM flux density distribution open circuit measurement on PM surfac e before and after 5250 hysteresis cycles.

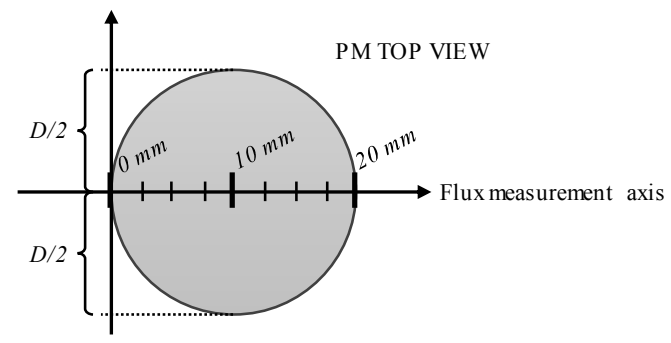

Fig. 12. Displacement line of the Gauss-meter on the PM sample surface.

\section{CONCLUSIONS}

Aging effects in $\mathrm{NdFeB} \mathrm{PMs}$ due to magnetization/demagnetization cycles have been analyzed in this paper. It has been shown that cycling has a direct impact on PM magnetic, electrical and thermal properties. $\mathrm{B}-\mathrm{H}$ and $\mathrm{J}-\mathrm{H}$ curves have been evaluated before and after a cycling process, showing that cycled magnets have lower magnetic energy, remanence and coercivity. Thermal behavior of PMs is also worsening in cycled magnets. Although the maximum working temperature of the magnet is not affected by aging, cycled magnets result in a poorer performance at higher temperatures due to the increase of the thermal coefficient of remanence. All these issues would have a negative impact on the torque capability of VF-PMSMs. The estimated PM high frequency resistance is seen to be also increased with cycling, which results in higher PM losses, higher demagnetization risk and in a reduction of the machine efficiency. Finally, it was shown that aging of PMs due to remagnetization results in a nonuniform magnetic field distribution, which in PMSMs may make the MMF waveform to change, inducing higher order harmonics in the machine airgap and consequently, in additional losses.

\section{REFERENCES}

[1] T. M. Jahns, "Flux-Weakening Regime Operation of an Interior Permanent-Magnet Synchronous Motor Drive," IEEE Trans. Ind. Appl., vol. IA-23, no. 4, pp. 681-689, July 1987. doi: 10.1109/TIA.1987.4504966

[2] Jang-Mok Kim and Seung-Ki Sul, "Speed control of interior permanent magnet synchronous motor drive for the flux weakening operation," IEEE Trans. on Ind. Appl., vol. 33, no. 1, pp. 43-48, Jan/Feb 1997. doi: 10.1109/28.567075

[3] K. Sakai, K. Yuki, Y. Hashiba, N. Takahashi and K. Yasui, "Principle of the variable-magnetic-force memory motor," IEEE ICEMS, pp. 1-6, Tokyo, 2009. doi: 10.1109/ICEMS.2009.5382812

[4] Z. Q. Zhu, Y. S. Chen and D. Howe, "Iron loss in permanentmagnet brushless $\mathrm{AC}$ machines under maximum torque per ampere and flux weakening control," IEEE Trans. on Mag., vol. 38, no. 5, pp. 3285-3287, Sep 2002. doi: 10.1109/TMAG.2002.802296

[5] V. Ostovic, "Memory motors-a new class of controllable flux PM machines for a true wide speed operation," IEEE Industry Applications Conference (IAS), vol.4, pp. 2577-2584, Chicago, IL, USA, 2001. doi: 10.1109/IAS.2001.955983

[6] V. Ostovic, "Memory motors," IEEE Industry Applications Magazine, vol. 9, no. 1, pp. 52-61, Jan/Feb 2003. doi: 10.1109/MIA.2003.1176459

[7] M. Ibrahim, L. Masisi, and P. Pillay, "Design of variable-flux permanent-magnet machines using alnico magnets," IEEE Trans. Ind. Appl. , vol. 51, no. 6, pp. 4482-4491, Nov./Dec. 2015. doi: 10.1109/TIA.2015.2461621

[8] A. Sun, J. Li, R. Qu, J. Chen and H. Lu, "Rotor design considerations for a variable-flux flux-intensifying interior permanent magnet machine with improved torque quality and reduced magnetization current," 2015 IEEE Energy Conversion Congress and Exposition (ECCE), Montreal, QC, 2015, pp. 784790. doi: 10.1109/ECCE.2015.7309769

[9] H. Liu, H. Lin, S. Fang and Z. Q. Zhu, "Permanent Magnet Demagnetization Physics of a Variable Flux Memory Motor," IEEE Trans. on Mag., vol. 45, no. 10, pp. 4736-4739, Oct. 2009. doi: 10.1109/TMAG.2009.2021408

[10] A. Athavale, K. Sasaki, B. S. Gagas, T. Kato and R. D. Lorenz, "Variable Flux Permanent Magnet Synchronous Machine (VFPMSM) Design Methodologies to Meet Electric Vehicle Traction Requirements with Reduced Losses," IEEE Trans. Ind. Appl., vol. 53, no. 5, pp. 4318-4326, Sept.-Oct. 2017. doi: 10.1109/TIA.2017.2701340

[11] H. Hua, Z. Q. Zhu, A. Pride, R. P. Deodhar and T. Sasaki, "A Novel Variable Flux Memory Machine With Series Hybrid Magnets," IEEE Trans. Ind. Appl., vol. 53, no. 5, pp. 4396-4405, Sept.-Oct. 2017. doi: 10.1109/TIA.2017.2709261

[12] L. Chang, T. R. Eastham and G. E. Dawson, "In-situ magnetization of $\mathrm{NdFeB}$ magnets for permanent magnet machines," in IEEE Transactions on Magnetics, vol. 27, no. 5, pp. 4355-4359, Sept. 1991.doi: 10.1109/20.105061

[13] Minna Haavisto, Sampo Tuominen, Timo Santa-Nokki, Harri Kankaanpää, Martti Paju, and Pekka Ruuskanen, "Magnetic Behavior of Sintered NdFeB Magnets on a Long-Term Timescale," Advances in Materials Science and Engineering, vol. 2014, 7 pages, Article ID 760584, 2014. doi:10.1155/2014/760584

[14] AllianceLCC: Design tools. "NdFeB Long Term Stability". Available online: http://www.allianceorg.com/pdfs/NdFeB_Long_term_stability.pdf

[15] D. Huger and D. Gerling, "The effects of thermal cycling on aging of Neodymium-Iron-Boron magnets," IEEE International Conference on Power Electronics and Drive Systems, pp. 389-392, Sydney, 2015. doi: 10.1109/PEDS.2015.7203464

[16] F. Fiorillo, "Characterization and Measurement of Magnetic Materials", Elsevier Academic Press, 1st Edition. 7th December 2004, eBook ISBN: 9780080528922,

[17] ABM Magnetics: Magnet properties. Available online: http://www.abm-magnetics.com/components/

[18] D. Fernandez, D. D. Reigosa, J. M. Guerrero, Z. Q. Zhu and F. Briz, "Permanent-Magnet Magnetization State Estimation Using HighFrequency Signal Injection," IEEE Trans. Ind. Appl., vol. 52, no. 4, pp. 2930-2940, July-Aug. 2016. doi: 10.1109/TIA.2016.2541616

[19] D. Díaz Reigosa, D. Fernandez, Z. Q. Zhu and F. Briz, "PMSM Magnetization State Estimation Based on Stator-Reflected PM Resistance Using High-Frequency Signal Injection," IEEE Trans. Ind. Appl., vol. 51, no. 5, pp. 3800-3810, Sept.-Oct. 2015. doi: 10.1109/TIA.2015.2437975

[20] D. F. Alonso, D. Reigosa, J. Guerrero, C. Suarez and F. Briz, "Impact of machine magnetization state on permanent magnet 
losses in permanent magnet synchronous machines," IEEE Energy Conversion Congress and Exposition (ECCE), Cincinnati, OH,
2017 ,

pp.

doi: 10.1109/ECCE.2017.8096967
5840-5845. 